\title{
MODELING UNCERTAINTY IN QUASI-HYDROSTATIC ISOTHERMAL SELF-GRAVITATING SLAB
}

\author{
Mohsen NeJad-Asghar \\ Department of Physics, Damghan University of Basic Sciences, Damghan, Iran \\ E-mail: nasghar@dubs.ac.ir \\ (Received September 15, 2006; Accepted January 8, 2007)
}

\begin{abstract}
The smoothed particle hydrodynamics (SPH) method is applied to construct the dispersion of fluctuations in quasi-hydrostatic configuration of an isothermal self-gravitating slab. The uncertainty of the implementation is evaluated, and a novel technique (acceleration error) is proposed to weaken this uncertainty. The two-fluid quasi-hydrostatic diffusion of small fluctuations is used to support the importance of the acceleration error. The results show that the uncertainty converges to a few percent by increasing of the SPH particle numbers. Considering the acceleration error weakens the uncertainty, and prohibits the serious dynamical consequences in slow dispersion of fluctuation in the quasi-hydrostatic evolution of the slab.
\end{abstract}

Key words : Hydrodynamics — methods: numerical — ISM: evolution

\section{INTRODUCTION}

The properties of molecular clouds are usually obtained from contour maps which highlight the densest optically thick structures. Contour maps show that the molecular clouds are highly fragmented, consisting several clumps and cores that make it somewhat arbitrary for any definition of them (Myers 1999). Studies of the time variability of absorption lines indicates the presence of fluctuations on scales of $10^{-4} p c$ (5-50 AU) and masses of $10^{-9} M_{\odot}$ (Boissé et al. 2005). Understanding of the origin and merging of these small fluctuations is of fundamental importance for a consistent theory of star formation and galactic evolution.

In molecular clouds, the velocity dispersion inferred from molecular line width is often larger than the gas sound speed inferred from transition temperatures (Solomon et al. 1987). Because of these turbulent motions, the global configuration of molecular clouds must be transient structures, and are probably dispersed after not much more than $10^{6} \mathrm{yr}$ (see, e.g. Larson 1981, Vázquez-Semadeni et al. 2005). Nejad-Asghar \& Ghanbari $(2003,2006)$ inferred that under certain conditions (e.g., expansion or contraction of the background), ambipolar drift heating in thermal instability may produce spherical, oblate, or prolate fluctuations. An important issue facing this senario is the dispersion of the newly formed fluctuations. In order to investigate this phenomena, we use the smoothed particle hydrodynamics (SPH) technique to implement the dispersion process in quasi-hydrostatic isothermal selfgravitating slab.

Many astrophysical dynamical processes have been studied numerically using SPH (Monaghan 1992). These include many stellar interaction processes such as relativistic calculations of compact binary mergers (e.g.,
Rasio et al. 2004), and stellar collisions (e.g., Freitag $\&$ Benz 2005), as well as modeling of accretion flows (e.g., Murray et al. 2004), supernova explosions (e.g., Garcia-Senz \& Bravo 2005), shock waves (e.g., NejadAsghar 2006), turbulence (e.g., Monaghan 2002), and also large-scale cosmological structure formation (e.g., Katz et al. 1996; Springel 2005), and galaxy formation (e.g., Steinmetz 1996; Sijacki \& Springel 2006).

The SPH method is basically to simulate the evolution of the field variables such as density, velocity, energy, etc. The variables are usually in the form of partial differential equations. The key idea of SPH is to substitute of integral interpolation with summation interpolation, and to calculate pressure gradient forces by kernel estimation, directly from the particle positions, rather than by finite differences on a grid. In this substitution, a truncation error introduce that normally depends on the disorder of the particles (Monaghan 1982). This error may be negligible in fast dynamical processes, however, must be considered in slow dispersion in quasi-hydrostatic fluids.

To simulate the diffusion process of fluctuations, we adapt the expressions for multi-phase flow derived by Monaghan \& Kocharyan (1995). In this paper the method is applied to the pioneer works of the isothermal self-gravitating slab with known solution to investigate the accuracy and convergence characteristics. In section 2 we present the basic formalism inherent to the presence of the fluctuations in a plane-parallel isothermal self-gravitating slab. In $\S 3$ the usual SPH equations for hydrodynamics are stated, the uncertainty is estimated, and a novel technique (acceleration error) is introduced. Dispersion of fluctuations is investigated to support the importance of the acceleration error. Finally, a summary of the paper and some prospects are given in section 4 . 


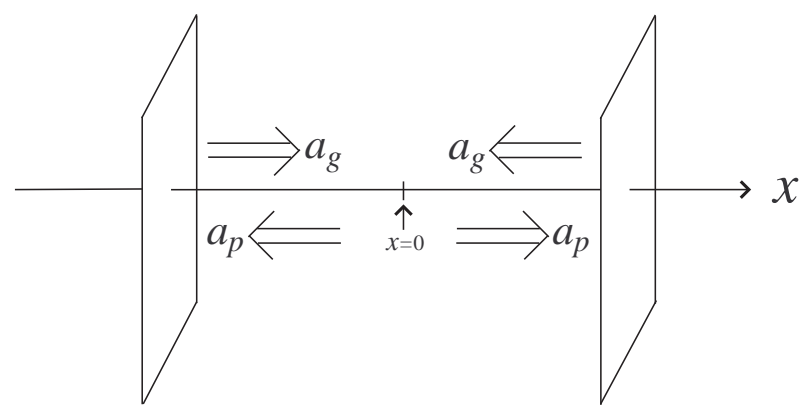

Fig. 1. - Schematic diagram of isothermal self-gravitating slab in $x$ direction.

\section{FORMULATION OF THE PROBLEM}

In this section, we modify the standard hydrostatic equilibrium of an isothermal plane-parallel self-gravitating slab to include the effects of fluctuations. The planar layer of molecular cloud material is spread in the $x$ direction (Fig. 1). The basic configuration of the mass conservation and the equation of motion take the form

$$
\begin{gathered}
\frac{\partial \rho}{\partial t}+\frac{\partial}{\partial x}(\rho v)=0 \\
\rho\left(\frac{\partial v}{\partial t}+v \frac{\partial v}{\partial x}\right)=-\frac{\partial p}{\partial x}+\rho g
\end{gathered}
$$

where $p=a^{2} \rho$ is the pressure ( $a$ is the isothermal sound speed) and $g$ is the gravitational field that obeys the poisson's equation

$$
\frac{\partial g}{\partial x}=-4 \pi G \rho
$$

If we denote the Lagrangian derivative by

$$
\frac{d}{d t} \equiv \frac{\partial}{\partial t}+v \frac{\partial}{\partial x}
$$

we can rewrite equations (1) and (2) as

$$
\begin{gathered}
\frac{d \rho}{d t}=-\rho \frac{\partial v}{\partial x}, \\
\frac{d v}{d t}=g-\frac{a^{2}}{\rho} \frac{\partial \rho}{\partial x} .
\end{gathered}
$$

Here, we introduce the fluctuations through the Ansatz

$$
\rho \rightarrow \rho(1+\eta)
$$

where the distribution of $\eta$ denotes the effect of fluctuations. Note that the fluctuations are assumed to have zero mean so that the density appearing in the equation of motion can be thought of as time-averaged quantity and play the same role in this generalized calculation.

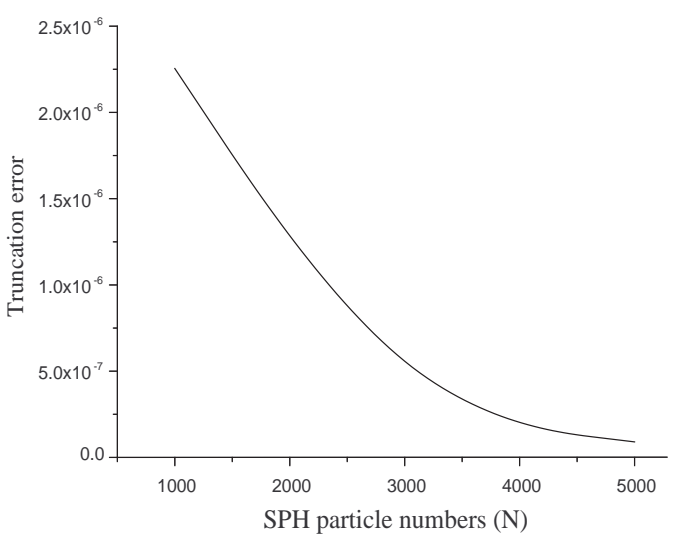

Fig. 2.- Truncation error of SPH simulation in different cases of particle numbers.

Following many previous treatments, a further simplification is possible if we introduce the surface density between mid-plane and $x>0$ as

$$
\sigma \equiv \int_{0}^{x} \rho\left(x^{\prime}, t\right) d x^{\prime}
$$

The usual defined surface density from $-x$ to $+x$ has twice the value of $\sigma$. By transformation from $(x, t)$ to $(\sigma, t)$

$$
\frac{d}{d t}=\left(\frac{\partial}{\partial t}\right)_{\sigma}, \quad \frac{\partial}{\partial x}=\rho\left(\frac{\partial}{\partial \sigma}\right)_{t}
$$

the field equation (3) can be integrated to give

$$
g=-4 \pi G \int_{0}^{x} \rho(1+\eta) d x^{\prime}
$$

while the equation of continuity (5) and the equation of motion (6) take the form

$$
\begin{gathered}
\frac{\partial x}{\partial \sigma}=\frac{1}{\rho(1+\eta)} \\
\frac{\partial^{2} x}{\partial t^{2}}=-4 \pi G \int_{0}^{x} \rho(1+\eta) d x^{\prime}-\frac{a^{2}}{1+\eta} \frac{\partial}{\partial \sigma}[\rho(1+\eta)] .
\end{gathered}
$$

A full solution to equations (10) and (11) is beyond the scope of this paper. We are interested in the hydrostatic equilibrium in the whole configuration of the slab. In this way, the left-hand side of equation (11) is zero, so that we may rewrite the force balance equation as

$$
4 \pi G \int_{0}^{x} \rho(1+\eta) d x^{\prime}+\frac{a^{2}}{1+\eta} \frac{\partial}{\partial \sigma}[\rho(1+\eta)]=0 .
$$


Following the work of Shu (1983), we introduce the non-dimension surface density $\tilde{\sigma}$, volume density $\tilde{\rho}$, and coordinate $\tilde{x}$ as

$$
\tilde{\sigma} \equiv \frac{\sigma}{\sigma_{\infty}}, \quad \tilde{\rho} \equiv \frac{a^{2}}{2 \pi G \sigma_{\infty}^{2}} \rho, \quad \tilde{x} \equiv \frac{2 \pi G \sigma_{\infty}}{a^{2}} x,
$$

where integration constant $\sigma_{\infty}$ is the value of $\sigma$ at $x=$ $\infty$ (where $\rho$ is zero). In this way, equations (10) and (12) become

$$
\begin{gathered}
\frac{\partial \tilde{x}}{\partial \tilde{\sigma}}=\frac{1}{\tilde{\rho}(1+\eta)}, \\
2 \int_{0}^{\tilde{x}} \tilde{\rho}(1+\eta) d \tilde{x}^{\prime}+\frac{1}{1+\eta} \frac{\partial}{\partial \tilde{\sigma}}[\tilde{\rho}(1+\eta)]=0 .
\end{gathered}
$$

In order to describe the average behaviour of the fluid, the method of intermediate time-averaging is used. In this method, we must average over an intermediate time-scale $\tau_{0}$ that obey the ordering

$$
0<\tau_{0} \ll \tau_{\text {diff }}
$$

where $\tau_{\text {diff }} \sim l / v_{d}$ is the diffusion time-scale required to disperse a fluctuation of drift velocity $v_{d}$, in the slab of length $l$ (i.e., diffusion crossing time). Let brackets denote time-averaging so that

$$
<\tilde{\rho}(1+\eta)>\equiv \frac{1}{\tau_{0}} \int_{0}^{\tau_{0}} \tilde{\rho}(1+\eta) d \tau^{\prime} \approx \tilde{\rho}
$$

where the errors of $O\left[\tau_{0} / \tau_{\text {diff } f}\right]$ are ignored.

Because the diffusion time-scale is also much shorter than the cloud time-scale, the slab is expected to evolve in a quasi-hydrostatic equilibrium state. The quasiequilibrium condition is found by integrating the timeaveraged force equation (15) to obtain

$$
\tilde{\rho}=1-\tilde{\sigma}^{2}
$$

where we have assumed that the fluctuations are relatively well behaved so that they obey constraint of the form

$$
\left\langle\frac{1}{1+\eta} \frac{\partial \eta}{\partial \tilde{\sigma}}\right\rangle \approx 0
$$

In order for this constraint to hold, the derivatives of the fluctuations must average to zero (as expected) and the derivatives must not be correlated with the fluctuations themselves. In other words, the fluctuations must be both spatially and temporally symmetric. Fluctuations, as expressed by equation (15), then diffuse in the quasi-equilibrium state that is given by the solution of equations (14) and (18), as follows (Spitzer 1942)

$$
\tilde{\rho}=\frac{1}{\cosh ^{2} \tilde{x}}, \quad \tilde{\sigma}=\tanh \tilde{x} .
$$

In this case, the gravitational acceleration profile, (9), is given by

$$
g(x)=-4 \pi G \sigma_{\infty} \tanh \left(\frac{2 \pi G \sigma_{\infty}}{a^{2}} x\right) .
$$

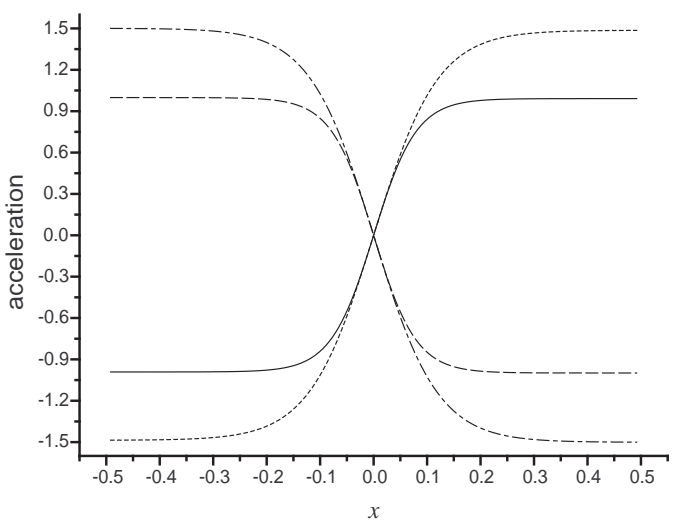

Fig. 3.- Acceleration of pressure gradient in the case of $a=0.2[v]$ (solid) and $a=0.3[v]$ (dot). Gravitational acceleration in the case of $a=0.2[v]$ (dash) and $a=0.3[v]$ (dash-dot).

\section{SPH CONSTRUCTION}

The chosen physical scales for length and time are $[l]=1 \mathrm{pc}$, and $[t]=1 \mathrm{Myr}$, respectively, so that velocity unit is approximately $[v]=1 \mathrm{~km} \mathrm{~s}^{-1}$. The gravity constant is set $G=1^{6}[\mathrm{~m}]^{-1}[l]^{3}[t]^{-2}$ for which the calculated mass unit is $[\mathrm{m}]=4.5 \times 10^{32} \mathrm{~kg}$. We assume that the slab is spread from $x=-0.5[l]$ to $x=+0.5[l]$ and the gas is represented by $N(=1000,3000,5000)$ discrete but smoothed SPH particles (i.e. Lagrangian sample points). The particles are overlapping, so that all the physical quantities involved can be treated as continues functions both in space and time. Overlapping is represented by the kernel function, $W_{\alpha \beta} \equiv$ $W\left(\left|\mathbf{r}_{\alpha}-\mathbf{r}_{\beta}\right|, h_{\alpha}\right)$, where $h_{\alpha}$ is the smoothing length of particle $\alpha$ (see, e.g., Monaghan 1992).

The basis of SPH is to approximate the integral interpolation

$$
A(\mathbf{r})=\int A\left(\mathbf{r}^{\prime}\right) W\left(\left|\mathbf{r}-\mathbf{r}^{\prime}\right|, h\right) d^{3} x^{\prime}
$$

by a summation interpolation

$$
A_{\alpha}=\sum_{\beta} \frac{m_{\beta}}{\rho_{\beta}} W\left(\left|\mathbf{r}_{\alpha}-\mathbf{r}_{\beta}\right|, h_{\alpha}\right)
$$

where the summation is over the nearest neighbor particles. In such substitution, a second order truncation error, $O\left(h_{\alpha}^{2}\right)$, is introduced that normally depends on the disorder of the particles (Monaghan 1982). We obtain the smoothing lengths so that the value of the number of the nearest neighbors, for each particle, are between $N_{\min }=4$ to $N_{\max }=6$. The truncation errors, $O\left(h_{\alpha}^{2}\right)$, are shown in Fig. 2. According to this figure, increasing of SPH particles cause to decrease the smoothing lengths, thus, the truncation errors decrease. 

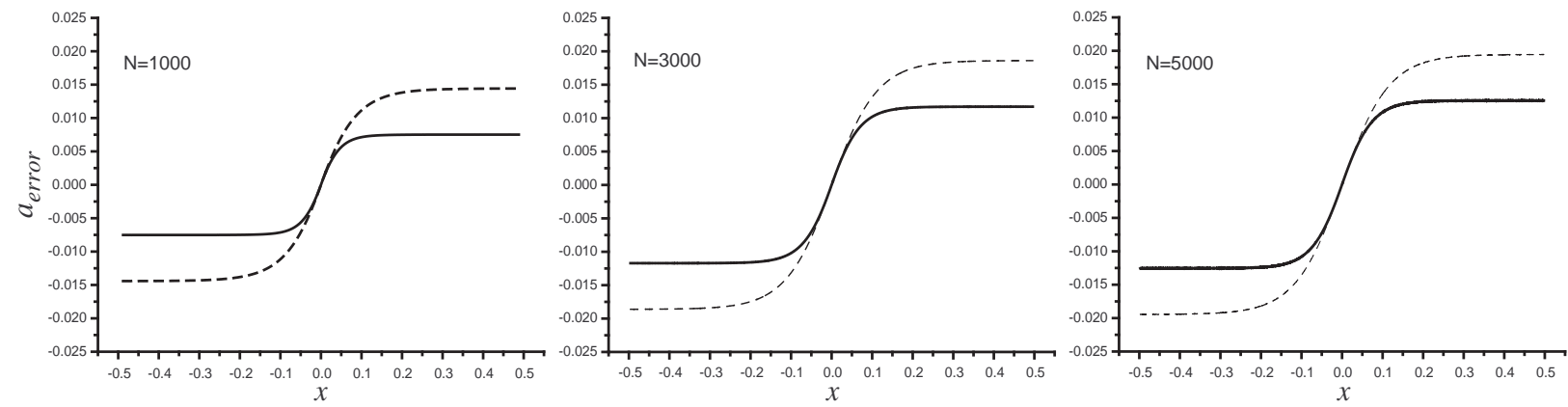

Fig. 4. - Acceleration error to set the SPH particles in the rest in the case of $a=0.2[v]$ (solid) and $a=0.3[v]$ (dot) for three instances of particle number.

Using the above idea, the density of particle $\alpha$ is estimated via usual summation over neighboring particles,

$$
\rho_{\alpha}=\sum_{\beta} m_{\alpha} W_{\alpha \beta},
$$

while the equation of motion is given by

$$
\frac{d \mathbf{v}_{\alpha}}{d t}=\mathbf{a}_{p r e, \alpha}+\mathbf{a}_{g r a v, \alpha}+\mathbf{a}_{v i s c, \alpha}
$$

where $\mathbf{a}_{p r e, \alpha}$ is the acceleration of pressure gradient

$$
\mathbf{a}_{p r e, \alpha}=-\sum_{\beta} m_{\beta}\left(\frac{p_{\alpha}}{\rho_{\alpha}^{2}}+\frac{p_{\beta}}{\rho_{\beta}^{2}}\right) \nabla_{\alpha} W_{\alpha \beta},
$$

and $\mathbf{a}_{\text {grav }, \alpha}$ is the gravitational acceleration of particle $\alpha$, which may be obtained by equation (9), as follows

$$
\begin{aligned}
\mathbf{a}_{\text {grav }, \alpha} & =-4 \pi \hat{i} G \sigma_{\alpha} \\
& \approx-4 \pi \hat{i} G \sum_{\beta} \rho_{\beta} ;\left(0<\left|x_{\beta}\right|<\left|x_{\alpha}\right|\right) .
\end{aligned}
$$

The artificial viscous acceleration, which includes a linear bulk viscosity component to prevent penetration as well as a Von Neumann-Richtmeyer type viscosity, is given by (Monaghan 1992)

$$
\mathbf{a}_{v i s c, \alpha}=-\sum_{\beta} m_{\beta} \Pi_{\alpha \beta} \nabla_{\alpha} W_{\alpha \beta},
$$

where

$$
\Pi_{\alpha \beta}= \begin{cases}\frac{\alpha^{*} \bar{a}_{\alpha \beta} \mu_{\alpha \beta}+\beta^{*} \mu_{\alpha \beta}^{2}}{\bar{\rho}_{\alpha \beta}}, & \text { if } \mathbf{v}_{\alpha \beta} \cdot \mathbf{r}_{\alpha \beta}<0 \\ 0, & \text { otherwise }\end{cases}
$$

is the artificial viscosity between particles $\alpha$ and $\beta$, where $\bar{\rho}_{\alpha \beta}=\frac{1}{2}\left(\rho_{\alpha}+\rho_{\beta}\right)$ is an average density, $\bar{a}_{\alpha \beta}=$ $\frac{1}{2}\left(a_{\alpha}+a_{\beta}\right)$ is the average sound speed, $\alpha^{*} \sim 1$ and $\beta^{*} \sim 2$ are the artificial coefficients, and $\mu_{\alpha \beta}$ is defined as its usual form

$$
\mu_{\alpha \beta}=-\frac{\mathbf{v}_{\alpha \beta} \cdot \mathbf{r}_{\alpha \beta}}{\bar{h}_{\alpha \beta}} \frac{1}{r_{\alpha \beta}^{2} / \bar{h}_{\alpha \beta}^{2}+10^{-3}}
$$

where $\bar{h}_{\alpha \beta}=\frac{1}{2}\left(h_{\alpha}+h_{\beta}\right)$. In quasi-equilibrium state, the artificial viscous acceleration is not important.

\section{(a) Uncertainty of SPH}

In time-averaged quasi-static equilibrium of the slab, the acceleration of pressure gradient must be approximately equal to the value of gravitational acceleration (Fig. 3). The small deviation (uncertainty) of the SPH method is widely acknowledged in the community. However, in slow dynamic evolution such as dispersion of fluctuations in the quasi-static configuration of the molecular clouds, this small deviation may cause to serious dynamical consequences.

To weaken the small deviation, and to set the SPH particles in quasi-rest state we propose a novel technique, acceleration error,

$$
\mathbf{a}_{\text {error }, \alpha} \equiv-\kappa\left(\mathbf{a}_{\text {pre }, \alpha}+\mathbf{a}_{\text {grav }, \alpha}\right),
$$

where $0 \leq \kappa \leq 1$ is a switch parameter. The acceleration error is shown in Fig. 4 in the case of $\kappa=1$. Since the slab is stable, the uncertainty provides perturbation with which the slab will oscillate around the equilibrium. The oscillation frequency,

$$
\nu_{o s c} \sim \frac{a}{\lambda}
$$

where $\lambda$ is the wavelength of induced perturbations, are shown in Fig. 5 according to different values of $\kappa$. It is obvious that the effect of acceleration error causes to weaken the oscillation, so that the value of oscillation frequency is inclined to zero.

The above uncertainty may be not only by the summation interpolation of pressure gradient, but also by 


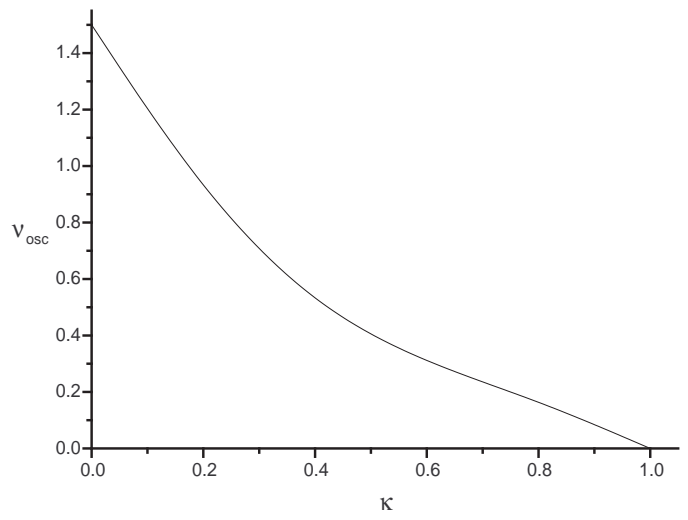

Fig. 5.- Oscillation frequency of the stable slab in the case of $a=0.3[v]$.

the particle distribution that dose not exactly mimic the gravitational acceleration profile (21). In this way, we define relative error of the gravitational acceleration,

$$
e_{g, \alpha} \equiv \frac{\left|a_{g r a v, \alpha}-g\left(x_{\alpha}\right)\right|}{\left|g\left(x_{\alpha}\right)\right|},
$$

and relative error of the acceleration of pressure gradient,

$$
e_{p, \alpha} \equiv \frac{\left|a_{p r e, \alpha}+g\left(x_{\alpha}\right)\right|}{\left|g\left(x_{\alpha}\right)\right|}
$$

that are plotted in Fig. 6 and Fig. 7. Increasing of the SPH particle number cause to increase the the relative error of the gravitational acceleration at high $|x|$, because the gravitational acceleration is estimated via summation in equation (27). The error converges ultimately to its value at the central region of the slab $(\sim 0.35 \%)$. On the other hand, relative error of the pressure acceleration is due to the summation interpolation, which is proportional to the second order truncation error, and converges to approximately $2.9 \%$. Thus, increasing and convergence of the error acceleration in Fig. 4 can be explained.

\section{(b) Diffusion of Fluctuations}

To investigate importance of the acceleration error in slow dynamic evolution, we consider the diffusion of uniform fluctuation

$$
\eta= \begin{cases}A \ll 1, & \text { if } 0 \leq \tilde{x} \leq A, \\ 0, & \text { otherwise, }\end{cases}
$$

in the quasi-static isothermal self-gravitating slab. For this purpose, we use the SPH implementation of the two-fluid technique as outlined by Monaghan \& Kocharyan (1995). Here, we refer to SPH particles of slab as $\alpha$ and $\beta$, and particles of fluctuations as $a$ and $b$. Likewise, for each SPH particle we must create two separate neighbor lists: one for neighbors of the same species and another for those of the different species. Consequently, each particle must have two different $h$-values.

The continuity equation for the SPH particles is given by its usual form (24), while the momentum equation is changed to include the drag force, which is experienced by the particles of slab due to collisions with the particles of fluctuation. The momentum equation for the particles of slab is

$$
\frac{d \mathbf{v}_{\alpha}}{d t}=\mathbf{a}_{p r e, \alpha}+\mathbf{a}_{g r a v, \alpha}+\mathbf{a}_{v i s c, \alpha}+\mathbf{a}_{d r a g, \alpha},
$$

and for the particles of fluctuation is

$$
\frac{d \mathbf{v}_{a}}{d t}=-\mathbf{a}_{d r a g, a},
$$

where the drag acceleration is (Monaghan \& Kocharyan 1995)

$$
\mathbf{a}_{d r a g, \alpha}=\gamma \sum_{b} m_{b} \frac{\mathbf{v}_{b \alpha} \cdot \mathbf{r}_{\alpha b}}{r_{\alpha b}^{2}+10^{-3} h_{\alpha b}^{2}} \mathbf{r}_{\alpha b} W_{\alpha b},
$$

where $\mathbf{r}_{\alpha b}$ is the position vector from $b$ to $\alpha\left(\equiv \mathbf{r}_{\alpha}-\mathbf{r}_{b}\right)$ and $\gamma$ is the coupling factor of two species

$$
\gamma \equiv \frac{\langle S v\rangle}{m}
$$

with $S$ being the collision cross-section, $m$ the particle mass, and $v$ relative velocity of two colliding species. Using the characteristic collision time-scale $\tau_{\text {coll }} \sim$ $1 /(\gamma \eta \rho)$, along with the approximation $\tau_{\text {coll }} \sim \tau_{\text {diff }}$, the value of $\gamma$ can be estimated. The pressure, gravitational potential, and viscous acceleration in the momentum equation of fluctuation is neglected since the fluctuation mass density is very less than the slab mass density $(\eta \rho \ll \rho)$. Note that the SPH particles of the slab undergo a drag force that weaken the hydrostatic equilibrium. If the drag acceleration is much greater than the error acceleration, we have a fast dynamical evolution that the fluctuation disperse rapidly. In this case, the effect of error acceleration is trampled by drag force. On the other hand, in slow dynamical evolution that we have $a_{\text {drag }} \ll a_{\text {error }}$, the effect of acceleration error can be predominated.

Here, we experiment the slow dispersion of a flat fluctuation (35), with $A \sim 0.01$. The initial drift velocity, assigned to each particle of fluctuation is $v_{d} \sim$ $0.001 a$. In this way, the dispersion time-scale is approximately obtained by $\tau_{\text {diff }} \sim \frac{10}{a}[t]$. Lack of the error acceleration in the simulating slab, causes an undesired expansion of it. Thus, the whole system of the fluctuation may be displaced, and a non-realistic velocity must be imposed to each SPH particles. Fig. 8(a) plots the velocity dispersion of the fluctuation at $\tau_{\text {diff }}$ for the case that the error acceleration is ignored. We see that the small uncertainty of SPH simulation cause 

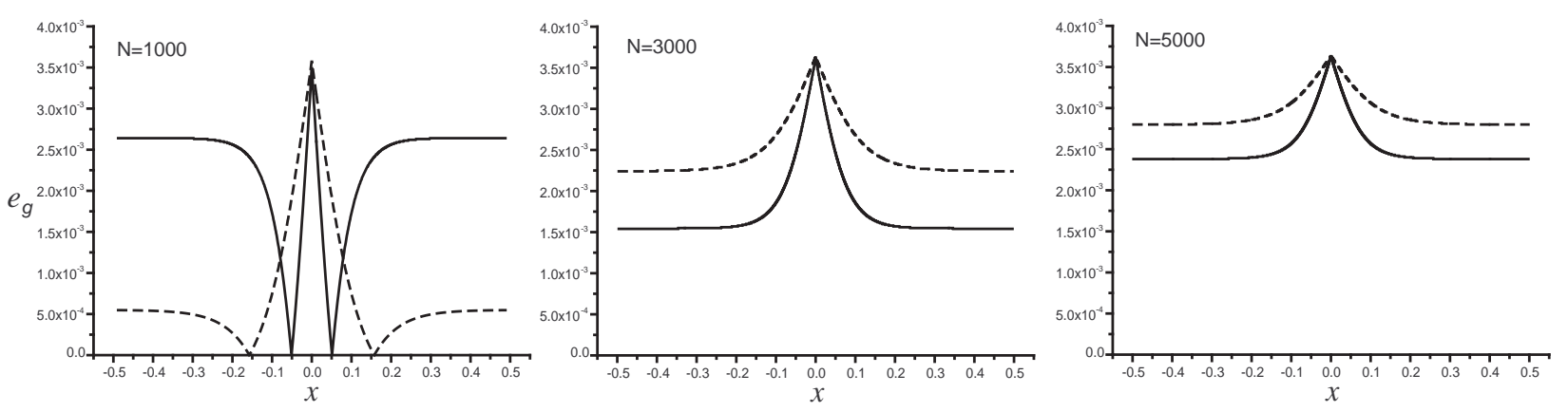

Fig. 6. - Gravitational acceleration error of SPH simulation in the case of $a=0.2[v]$ (solid) and $a=0.3[v]$ (dot) for three instances of particle numbers.
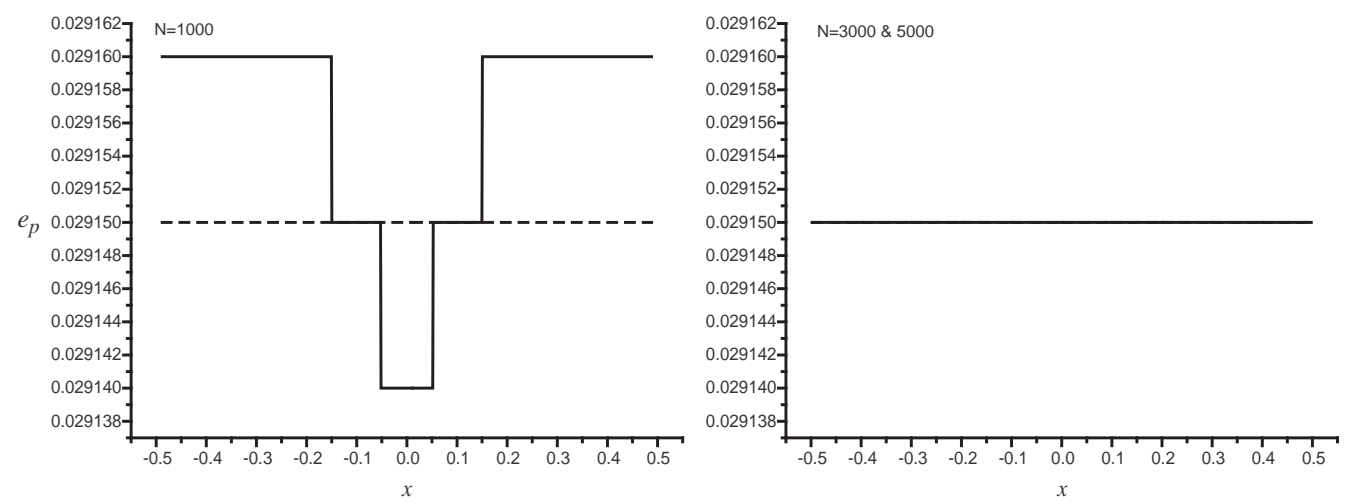

Fig. 7.- Error of summation interpolation in the acceleration of pressure gradient of SPH simulation in the case of $a=0.2[v]$ (solid) and $a=0.3[v]$ (dot) for three instances of particle numbers.

to prevent the dispersion of fluctuation even at $\tau_{\text {diff }}$. But, including the acceleration error weaken the uncertainty, thus, evolution of the slab and dispersion of the fluctuations may be thoroughly realistic. This case is shown in Fig. $8(\mathrm{~b})$ at times $0.01 \tau_{\text {diff }}$ and $0.03 \tau_{\text {diff } f}$. According to this figure, the small drag acceleration contract the slab, and prohibit the undesired displacement of the fluctuation whole place. It is obvious that handling the error acceleration not only cause a desired small contraction of the density profile but also gives a correct simulation for slow dispersion of fluctuation.

\section{SUMMARY AND PROSPECTS}

The SPH method was used to construct the dispersion of fluctuations in hydrostatic configuration of the isothermal self-gravitating slab. In the case of timeaveraged quasi-static equilibrium of the slab, the acceleration of pressure gradient must be set equal approximately to the value of gravitational acceleration. The deviation is due to the tolerance of the computational techniques. The tolerance exists be not only because of the summation interpolation of pressure gradient, but also due to the particle distribution that dose not ex- actly mimic the equilibrium profile. The Figs. 5 and 6 show the estimated uncertainty of SPH particles, and indicate that results converge to within a few percent accuracy if the number of particles exceeds a few thousands.

In slow dynamic evolution case, such as dispersion of fluctuations in the quasi-static configuration of the molecular clouds, this small uncertainty may cause a serious dynamical consequence. To weaken this small deviation and to set the SPH particles in quasi-rest state, we proposed a novel technique entitled error acceleration. The two-fluid quasi-hydrostatic diffusion of small fluctuations in a slab was used to support the importance of the acceleration error. The SPH particles of the slab undergo a drag force that weakens the hydrostatic equilibrium. If the drag acceleration is much greater than the acceleration error, we have a fast dynamical evolution that the fluctuation disperse rapidly. In this case, the effect of acceleration error is trampled by drag force. On the other hand, in slow dynamical evolution in which we have $a_{d r a g} \ll a_{\text {error }}$, the effect of acceleration error predominates.

The results show that the lack of acceleration error 




(a)



(b)

Fig. 8. - Velocity dispersion of the fluctuation in the quasi-hydrostatic slab (a) without the acceleration error, (b) for the case that the acceleration error is considered.

in the simulation, causes an undesired expansion of the slab. This phenomena displaces the whole system of the fluctuation, and a non-realistic velocity is imposed to each SPH particle. While, considering the acceleration error not only causes a desired small contraction of the density profile but also gives a correct simulation for slow dispersion of fluctuation. The results of this paper may be applied to investigate the subsonic drift velocity and ambipolar diffusion heating rate of a lightly ionized isothermal self-gravitating slab.

\section{ACKNOWLEDGEMENTS}

I gratefully acknowledge the comments of an anonymous referee that helped to clarify an earlier draft of this paper. This work was supported by the grant number 3159 of the Research Council of the Damghan University of Basic Sciences.

\section{REFERENCES}

Boissé, P., Le Petit, F., Rollinde, E., Roueff, E., Pineau des Forêts, G., Andersson, B. G., Gry, C., \& Felenbok, P., 2005, A far UV study of interstellar gas towards HD 34078: High excitation H2 and small scale structure, Astron. Astrophys., 429, 509

Freitag, M. \& Benz, W., 2005, A Comprehensive Set of Simulations of High-Velocity Collisions Between Main-Sequence Stars, Mon. Not. R. Astron. Soc., 358,1133

Garcia-Senz, D. \& Bravo, E., 2005, Type Ia Supernova Models Arising from Different Distributions of Igniting Points, Astron. Astrophys., 430, 585

Katz, N., Weinberg, D. H., \& Hernquist, L., 1996, Cosmological Simulations with TreeSPH, Astrophys. J. Suppl., 105, 19

Larson, R. B., 1981, Turblence and Star Formation in Molecular Clouds, Mon. Not. R. Astron. Soc., 194, 809
Monaghan, J. J., 1982, Why Particle Methods Work?, SIAM J. Sci. Stat. Comput., 3, 422

Monaghan, J. J., 1992, Smoothed Particle Hydrodynamics, Annu. Rev. Astron. Astrophys, 30, 543

Monaghan, J. J., 2002, SPH Compressible Turbulence, Mon. Not. R. Astron. Soc., 335, 843

Monaghan, J. J. \& Kocharyan, A., 1995, SPH simulation of multi-phase flow, Comput. Phys. Commun., 87,225

Murray, J. R., Truss, M. R., Foulkes, S. B., Haswell, C. A., \& Manson, K. J., 2004, Hydrodynamic Modelling of Accretion Flows, Revista Mexicana de Astronomia y Astrofisica (RMXAA), 20, 166

Myers, P. C., 1999, The Physics and Chemistry of the Interstellar Medium, Proceedings of the 3rd Cologne-Zermatt Symposium, edited by Ossenkopf, V., Stutzki, J., and Winnewisser, G., GCA-Verlag Herdecke, p. 227

Nejad-Asghar, M., 2006, Simulation of Shock Waves by Smoothed Particle Hydrodynamics, in proceeding of 10th Asrtonomy Meeting in Iran, edited by Khakian, M., astro-ph/0601480

Nejad-Asghar, M., \& Ghanbari, J., 2003, Linear thermal instability and formation of clumpy gas clouds including ambipolar diffusion, Mon. Not. R. Astron. Soc., 345, 1323

Nejad-Asghar, M. \& Ghanbari, J., 2006, Formation of Small-Scale Condensations in the Molecular Clouds via Thermal Instability, Astrophys. Space Sci., 302, 243

Rasio, F., Faber, J., Kobayashi, S., \& Laguna, P., 2004, Relativistic SPH Calculations of Compact Binary Mergers, in proceeding of JGRG14, edited by Hikida, W., Sasaki, M., Tanaka, T., and Nakamura, T., astro-ph/0503007

Shu, F., 1983, Ambipolar Diffusion in Self-Gravitating Isothermal Layers, Astrophys. J., 273, 202 
Sijacki, D., \& Springel, V., 2006, Physical Viscosity in Smoothed Particle Hydrodynamics Simulations of Galaxy Clusters, Mon. Not. R. Astron. Soc., tmp, 868

Solomon, P. M., Rivolo, A. R., Barrett, J., \& Yahil, A., 1987, Mass, luminosity, and line width relations of Galactic molecular clouds, Astrophys. J., 319, 730

Spitzer, L., 1942, Physics of Interstelar Medium, Astrophys. J., 95, 329

Springel, V., 2005, The cosmological simulation code GADGET-2, Mon. Not. R. Astron. Soc., 364, 1105

Steinmetz, M., 1996, GRAPESPH: cosmological smoothed particle hydrodynamics simulations with the special-purpose hardware GRAPE, Mon. Not. R. Astron. Soc., 278, 1005

Vázquez-Semadeni, E., Kim, J., Shadmehri, M., Ballesteros-Paredes, J., 2005, The Lifetimes and Evolution of Molecular Cloud Cores, Astrophys. J., 618,344 